\title{
Giant nonlinear interaction between two optical beams via a quantum dot embedded in
} a photonic wire

Nguyen, H.A.; Grange, T.; Reznychenko, B.; Yeo, I. ; de Assis, P.L.; Tumanov, D.; Fratini, F.; Malik, N. S.; Dupuy, E.; Gregersen, Niels

Total number of authors:

14

Published in:

Physical Review B (Condensed Matter and Materials Physics)

Link to article, DOI:

10.1103/PhysRevB.97.201106

Publication date:

2018

Document Version

Publisher's PDF, also known as Version of record

Link back to DTU Orbit

Citation (APA):

Nguyen, H. A., Grange, T., Reznychenko, B., Yeo, I., de Assis, P. L., Tumanov, D., Fratini, F., Malik, N. S., Dupuy, E., Gregersen, N., Auffeves, A., Gérard, J-M., Claudon, J., \& Poizat, J-P. (2018). Giant nonlinear interaction between two optical beams via a quantum dot embedded in a photonic wire. Physical Review $B$ (Condensed Matter and Materials Physics), 97, [201106]. https://doi.org/10.1103/PhysRevB.97.201106

\section{General rights}

Copyright and moral rights for the publications made accessible in the public portal are retained by the authors and/or other copyright owners and it is a condition of accessing publications that users recognise and abide by the legal requirements associated with these rights.

- Users may download and print one copy of any publication from the public portal for the purpose of private study or research.

- You may not further distribute the material or use it for any profit-making activity or commercial gain

- You may freely distribute the URL identifying the publication in the public portal 


\title{
Giant nonlinear interaction between two optical beams via a quantum dot embedded in a photonic wire
}

\author{
H. A. Nguyen, ${ }^{1}$ T. Grange, ${ }^{1}$ B. Reznychenko, ${ }^{1}$ I. Yeo,${ }^{1,2}$ P.-L. de Assis, ${ }^{3}$ D. Tumanov, ${ }^{1}$ F. Fratini, ${ }^{1}$ N. S. Malik, ${ }^{2}$ E. Dupuy,${ }^{2}$ \\ N. Gregersen, ${ }^{4}$ A. Auffèves, ${ }^{1}$ J.-M. Gérard, ${ }^{2}$ J. Claudon, ${ }^{2}$ and J.-Ph. Poizat ${ }^{1, *}$ \\ ${ }^{1}$ Université Grenoble Alpes, CNRS, Grenoble INP, Institut NEEL, "Nanophysique et Semiconducteurs" group, F-38000 Grenoble, France \\ ${ }^{2}$ Université Grenoble Alpes, CEA, INAC, PHELIQS, "Nanophysique et Semiconducteurs” group, F-38000 Grenoble, France \\ ${ }^{3}$ Gleb Wataghin Institute of Physics, University of Campinas - UNICAMP, 13083-859, Campinas, São Paulo, Brazil \\ ${ }^{4}$ Department of Photonics Engineering, DTU Fotonik, Kongens Lyngby, Denmark
}

(Received 18 June 2017; revised manuscript received 23 April 2018; published 11 May 2018)

\begin{abstract}
Optical nonlinearities usually appear for large intensities, but discrete transitions allow for giant nonlinearities operating at the single-photon level. This has been demonstrated in the last decade for a single optical mode with cold atomic gases, or single two-level systems coupled to light via a tailored photonic environment. Here, we demonstrate a two-mode giant nonlinearity with a single semiconductor quantum dot (QD) embedded in a photonic wire antenna. We exploit two detuned optical transitions associated with the exciton-biexciton QD level scheme. Owing to the broadband waveguide antenna, the two transitions are efficiently interfaced with two free-space laser beams. The reflection of one laser beam is then controlled by the other beam, with a threshold power as low as 10 photons per exciton lifetime $(1.6 \mathrm{nW})$. Such a two-color nonlinearity opens appealing perspectives for the realization of ultralow-power logical gates and optical quantum gates, and could also be implemented in an integrated photonic circuit based on planar waveguides.
\end{abstract}

DOI: 10.1103/PhysRevB.97.201106

Whether classical or quantum, optical communication has proven to be the best approach for long-distance information distribution. All-optical data processing has therefore raised much interest in recent years, as it would avoid energy- and coherence-consuming optics-to-electronics conversion steps [1]. To enable photon-photon interactions, low-power optical logic faces the challenge of implementing nonlinear effects that usually occur at high power. Interestingly, giant optical nonlinearities, ultimately operating at the single-photon level, can be achieved via resonant interactions with systems featuring discrete energy levels [2]. While atomic gas [3,4], single atoms [5-12], and molecules [13,14] have enabled remarkable achievements, solid-state systems are currently being actively investigated to realize integrated devices. Similarly to their atomic counterpart, most solid-state realizations are based on the concept of a "one-dimensional atom" [15], wherein a single atomiclike system is predominantly coupled to a single propagating spatial mode. Such a preferential coupling has first been obtained via a resonant interaction with a microcavity, enabling the demonstration of single-mode giant nonlinearities [16-24]. However, practical optical computing requires a nonlinear interaction between two different optical channels, with few demonstrations up to now $[11,12,14,25,26]$. In this context, one-dimensional atoms based on waveguides are particularly appealing. While they can be operated in the single-mode regime [27], they also enable nonlinear interactions between two optical fields having different colors, as proposed in Refs. [28,29].

\footnotetext{
*Corresponding author: jean-philippe.poizat@neel.cnrs.fr
}

In this Rapid Communications, we demonstrate this very idea with a semiconductor quantum dot (QD) embedded in a tapered waveguide antenna [Fig. 1(a)]. As proposed in Ref. [29], we exploit two spectrally detuned QD transitions, associated with the biexciton-exciton ladderlike level scheme. Owing to the broadband antenna, both transitions are efficiently interfaced with free-space optical beams. A first laser beam, resonant with one transition, is used to control the reflection of a second probe beam, tuned on the other transition. We explore two different configurations, and discuss their respective potentials for ultralow-power optical computation and for photonic quantum computation. Such giant two-color nonlinearities, which appear for a control power as small as $1 \mathrm{nW}$, could also be implemented in an integrated photonic circuit based on planar waveguides.

As shown in Fig. 1(a), the device under consideration is a vertical GaAs photonic wire antenna which embeds a self-assembled InAs QD near its base. In such a high-index waveguide, a large fraction of the QD spontaneous emission is funneled into the fundamental guided mode [33]. Note that we exploit here an open waveguide geometry: There is no bottom mirror and the top facet reflectivity is suppressed by antireflection coating. In the following, the device is addressed via the top port which is engineered for efficient out- and in-coupling. As it propagates along the conical taper towards the top facet, the fundamental guided mode undergoes a lateral expansion. The antenna output beam, with a nearly ideal Gaussian transverse profile, is directive and can be efficiently collected by free-space optics [34,35]. Reversibly, focusing a mode-matched Gaussian beam on the top facet of the photonic wire leads to a large interaction cross section with the QD. Crucially, the broad operation bandwidth of 
(a)
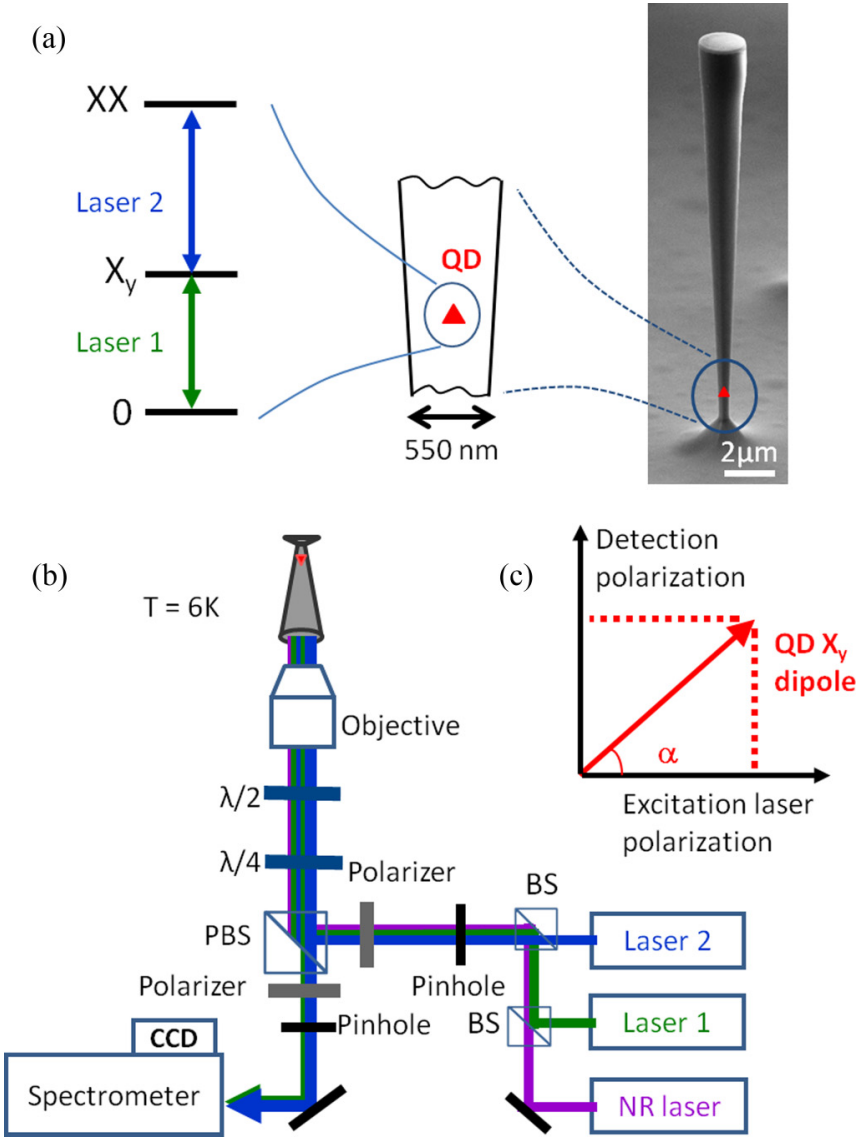

FIG. 1. Sample and setup. (a) A scanning electron microscope image of the photonic wire antenna is shown on the right. The center part details the location of the QD at the bottom of the wire. The waveguide, with a local diameter of $500 \mathrm{~nm}$, ensures an efficient coupling of the QD spontaneous emission to the fundamental guided mode. The empty QD (0), the excitonic $\left(X_{y}\right)$, and biexcitonic $(X X)$ states form a nondegenerate three-level ladder scheme as represented on the left. The two transitions are addressed by two different lasers. (b) Experimental setup. Two tunable continuous-wave lasers excite the QD transitions. The lasers are spatially filtered with a pinhole located at the focal point of a lens and focused on the device with a microscope objective. A confocal detection with a pinhole selects only the light coming out of the photonic wire. An extra nonresonant (NR) laser is used for photoluminescence spectroscopy, and as a "quietening laser" during the experiments [30,31]. A polarizing beam splitter (PBS) is used for a cross-polarized detection scheme. (c) The lasers are linearly polarized at an angle $\alpha=27^{\circ}$ with respect to the QD dipoles of interest. The detection is performed along the polarization orthogonal to the laser polarization. Wave plates in front of the objective ensure a precise control of the laser polarization [32].

the antenna enables an efficient interfacing of several detuned optical transitions hosted by the QD.

The device is attached to the cold finger of a liquid helium cryostat $(T=6 \mathrm{~K}$ ) with an optical access. As shown in Fig. 1(b), excitation laser beams are focused on the top facet of the antenna with a microscope objective (numerical aperture 0.4). The same objective is used to collect the QD emission, which is then sent to a grating spectrometer equipped with a $\mathrm{CCD}$ camera for spectral analysis (spectral resolution $12 \mu \mathrm{eV}$ ).
We focus on an individual, spectrally isolated QD, which is first characterized by performing standard photoluminescence measurements. Using nonresonant laser excitation, we identify the transitions associated with the recombination of the neutral exciton $(X)$ and biexciton $(X X)$. The corresponding transition energies, around $1.36 \mathrm{eV}$, are separated by $0.6 \mathrm{meV}$. We have measured lifetimes of $1.4 \mathrm{~ns}(0.7 \mathrm{~ns})$ for the excitonic (biexcitonic) level. Owing to the nonperfect QD circular symmetry, the excitonic level features a fine structure splitting of $25 \mu \mathrm{eV}$. The two bright excitons, denoted $X_{x}$ and $X_{y}$, feature two orthogonal linear optical dipoles oriented along the crystallographic axis $x=$ [110] and $y=[1 \overline{1} 0]$ of GaAs [36].

In the following, we drive resonantly the $0 \leftrightarrow X_{y}$ (" 0 " is the QD ground state) and $X_{y} \leftrightarrow X X$ transitions with two continuous-wave external grating diode lasers. We employ a cross-polarization scheme for excitation and detection [32]. Specifically, the two excitation lasers feature a linear polarization, oriented with an angle $\alpha=27^{\circ}$ with respect to the $y$-optical dipole [see Fig. 1(c)]. Owing to a polarizing beam splitter, we collect on the detection path the light with a polarization perpendicular to the one of the lasers. Laser parasitic reflections are then suppressed by a factor of $10^{-4}$, while a large fraction of the QD signal is detected by our setup. We investigate in this work a cross nonlinear effect which is revealed by measuring the reflectivity of one of the laser beams (probe beam) as a function of the intensity of the other one (control beam). We discuss below the two scenarios corresponding to the control laser being tuned either around the upper or the lower transition of the three-level scheme. In our experiments, the main qualitative effects are explained by considering the three-level ladder formed by $0, X_{y}$, and $X X$. However, as discussed in the Supplemental Material [30], full quantitative modeling [37,38] requires the inclusion of both excitonic levels.

We first consider the case in which the control (probe) laser is tuned on the lower (upper) transition (see Fig. 2). We will refer to this configuration as the "population switch," since the physics at work in this situation is the control of the $X_{y}$ state population by the control laser. When the latter is off [Fig. 2(a)], both $X_{y}$ and $X X$ states are empty so that the probe laser beam sees a transparent medium and is totally transmitted. As the control laser intensity is increased towards saturation of the lower transition, the $X_{y}$ state becomes populated and the probe beam experiences a dipole-induced reflection [Fig. 2(b)].

This population switch mechanism is evidenced in Fig. 2(c), which shows the probe laser reflectivity as a function of the control laser power. The switching threshold is only $1.6 \mathrm{nW}$ or 10 photons/lifetime. Here, "lifetime" refers to the QD excitonic lifetime, whose measured value is $1.4 \mathrm{~ns}$. The probe reflectivity reaches a maximum for a control laser power as low as $16 \mathrm{nW}$ (100 photons/lifetime). Increasing further the control laser power leads to an Autler-Townes splitting [39-42] of the intermediate state, which brings the probe beam out of resonance and reduces its reflectivity [Fig. 2(c)].

This experimental behavior is well fitted with our model over four orders of magnitude of control laser power, for two different probe powers (see Fig. 2(c) and Supplemental Material [30]). The values of the reflectivity $R$ and the switching power $P_{s}$ are presently limited by imperfections of our system, and are fully accounted for by our model. The parameters 
(a)

Control off
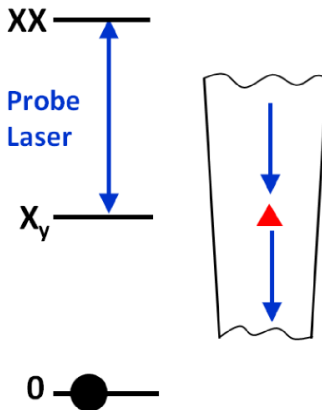

(b)

Control on

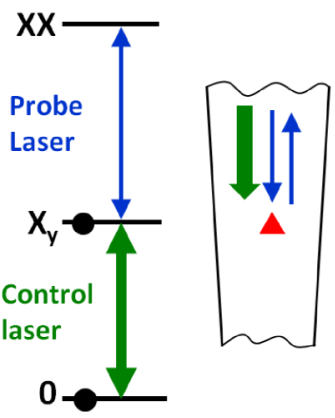

(c)

Control laser power $(\mathrm{nW})$

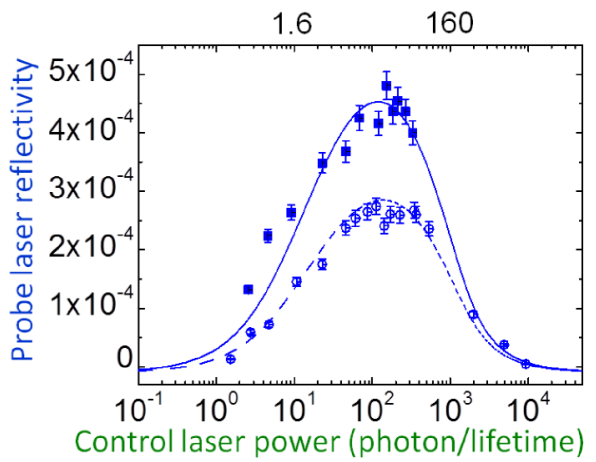

FIG. 2. Population switch. The control (probe) laser is tuned around the lower (upper) transition. (a) When the control laser is off, the probe laser beam sees empty levels and is transmitted. (b) When the control laser is on, the excitonic state is populated so that the probe laser beam is reflected. (c) Probe reflectivity as a function of the control laser power, for a probe laser power of $0.5 \mathrm{nW}$ (solid squares) and of $2.6 \mathrm{nW}$ (open circles). This corresponds respectively to 0.1 and 0.5 of the saturation power. The solid and dashed lines are fits using our theoretical model with parameters that have also been used for the fitting of the data presented in Fig. 3.

that are affecting the performances are the fraction $\varepsilon$ of input light coupled to the QD, and the linewidth broadening. The quantity $\varepsilon$ is the product of the mode matching efficiency, the taper modal efficiency, and the waveguide coupling efficiency $\beta$. From the global fitting of our experimental results, we extract $\varepsilon=0.26 \pm 0.01$, which is in line with a Fourier modal method calculation [43] based on the sample geometry. Here, $\varepsilon$ is essentially limited by the $\beta$ factor: The waveguide diameter $(500 \mathrm{~nm})$ is two times larger than the one offering an optimal transverse confinement. In our experiment, the measured linewidth $\Gamma$ is broadened to $\Gamma=10 \gamma$, where $\gamma$ is the lifetime-limited linewidth. This broadening is shared between the homogeneous origin due to pure dephasing, and the inhomogeneous origin caused by spectral diffusion [30].

With ideal parameters $(\varepsilon=1$ and $\Gamma=\gamma)$, losses are vanishing, so that all input light is used to saturate the QD, and the cross-polarized detection scheme can be removed by aligning the laser polarizations to the exciton dipole direction and detecting the reflected light along this polarization as well. In this case, based on our theoretical model, we find that the switching power can be as low as $P_{s}^{(0)}=0.1$ photons/lifetime but that the maximum reflectivity can never exceed $R^{(0)}=0.1$. This limitation comes from the partial population of the $X_{y}$ state at low control powers and the Autler-Townes induced probe laser detuning for higher control powers (see below). It is also due to the population leaks caused by the presence of the other fine structure split level $X_{x}$, which is populated via spontaneous emission from the biexcitonic $(X X)$ state [30].

To overcome this fundamental limitation, we explore another switch mechanism in which the control (probe) laser is tuned on the upper (lower) transition (see Fig. 3). The physical effect here is the dressing of the upper transition by the control laser when it is well above saturation. In the absence of the control laser [Fig. 3(a)], the weak probe beam (below saturation) is reflected when on resonance with the lower transition [16]. When the control laser is turned on above saturation [Figs. 3(b) and 3(c)], the $X_{y}$ state splits into two dressed states, as a result of the Autler-Townes effect [39-42]. The probe beam is then no longer resonant and its reflection switched off. In this "Autler-Townes" configuration, the switching threshold is found to be around $200 \mathrm{nW}$ [cf. Fig. 3(d)]. Note that, owing to the population of the other fine structure split excitonic level by the control laser via the relaxation of the $X X$ state, the reflectivity exhibits a local maximum for nonvanishing control power (see Ref. [30] for details). The Autler-Townes splitting effect is well evidenced in Figs. 3(e)-3(g), exhibiting the typical anticrossing of the probe laser reflectivity as a function of the two laser detunings. Using the same set of parameters for all the data presented in this work, our theoretical model is able to quantitatively reproduce all the experimental results (Figs. 2 and 3).

Interestingly, and contrary to the population switch configuration, the Autler-Townes configuration potentially shows perfect performances (i.e., $R^{(0)}=1, \quad P_{s}^{(0)}=1$ photons/lifetime) with ideal parameters $(\varepsilon=1$ and $\Gamma=\gamma)$ in the copolarized setting. Moreover, our theoretical model indicates that, in this case, the reflectivity is almost fully coherent, which is a key feature in the perspective of the realization of quantum logical gates [30]. For these two reasons, the AutlerTownes configuration is the better choice for a logical gate in both classical and quantum contexts with state-of-the-art devices allowing copolarized operation, as discussed below.

Using models developed by some of us in Ref. [24], we have also theoretically investigated the pulsed situation for both configurations. We have found that the pulsed regime leads to similar performances for the reflectivity switching threshold and our model predicts a pulse bandwidth of a few $10 \mathrm{MHz}$, set by the QD lifetime.

Let us mention that combining state-of-the-art optical coupling with a narrow line QD would allow us to come rather close to these ideal parameters and to implement an efficient ultralow-power all-optical switch. Optical couplings as high as $\varepsilon=0.75$ have already been reported in slightly narrower waveguides and expected values for optimized designs are as high as $\varepsilon=0.95$ [34]. Additionally, close to lifetimelimited linewidths (i.e., $\Gamma \approx \gamma$ ) have been obtained recently by applying a voltage bias across the QD $[44,45]$. Suitable electrical contacts can be implemented on our photonic wire, without degrading the optical properties, using the designs proposed by some of us in Ref. [46]. 
(a) Control off

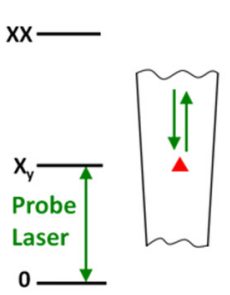

(b) Control on

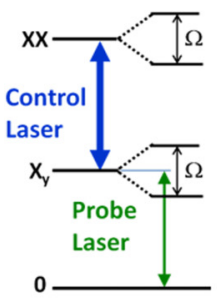

(c)

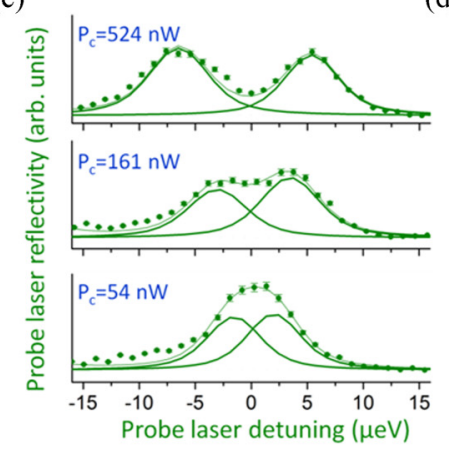

(d)
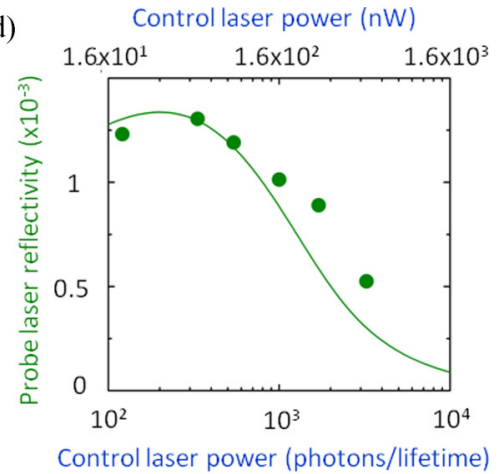

(e)

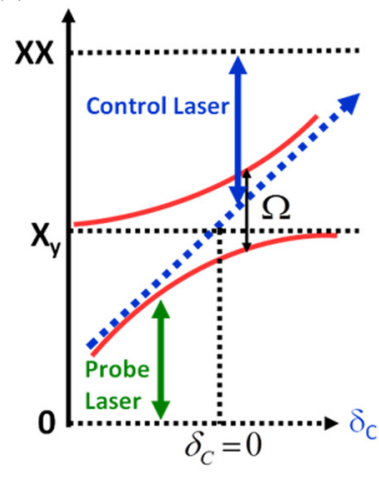

(f)

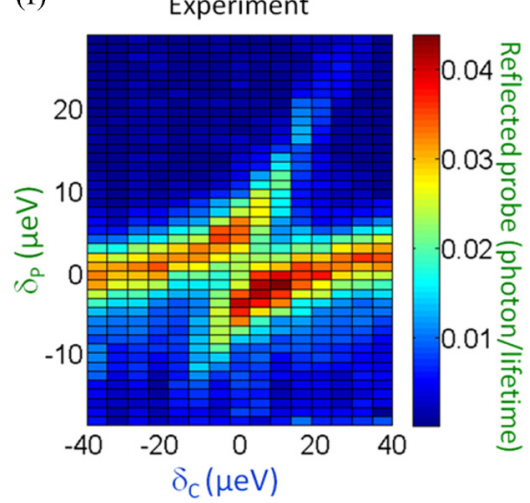

(g)

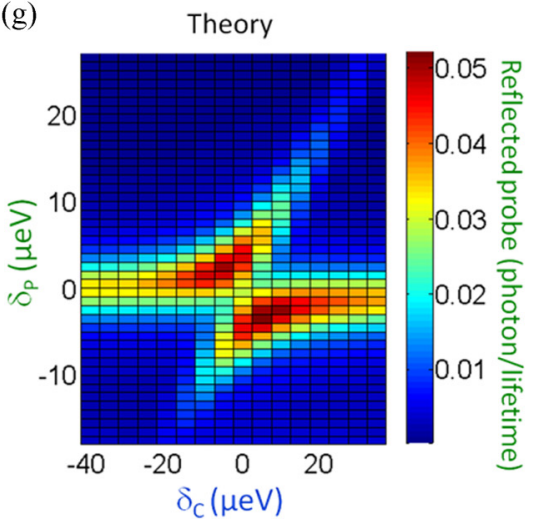

FIG. 3. Switch in the Autler-Townes configuration. The control (probe) laser is tuned on the upper (lower) transition. (a) When the control laser is off, the probe is reflected. (b) When the control laser is on, it splits the $X_{y}$ state, so that the probe is no longer on resonance, and therefore transmitted. (c) Probe reflectivity as a function of its detuning for different control laser powers, and a zero control laser detuning. The solid lines are fits using an individual line profile with the line position as a free parameter. The thinner line is the sum of the individual lines. We have checked that the splitting scales as the square root of the control laser power (data not shown). (d) Probe reflectivity as a function of the control laser power. The probe laser power is $1 \mathrm{nW}$. The solid line is the result given by our theoretical model. (e) Position of the Autler-Townes doublet as the control laser is scanned across the upper transition. (f) Experimental [30] and (g) theoretical reflectivity of the probe laser beam as a function of probe and control laser detunings. The probe (control) laser power is $1 \mathrm{nW}(274 \mathrm{nW})$. The theoretical fits are all made with the same set of parameters as in Fig. 2(c).

In conclusion, we have experimentally demonstrated a giant two-mode cross nonlinearity between two different laser beams in a semiconductor QD embedded in a photonic wire. This nonlinearity appears at an optical power as low as 10 photons per emitter lifetime. We have identified the Autler-Townes configuration as a promising configuration for the realization of classical, as well as quantum, ultralow-power logical gates. Importantly, our results can be readily transferred to planar GaAs photonic chips based on photonic crystal geometries [27] or ridge waveguides $[47,48]$, and therefore offer interesting perspectives for on-chip photonic computation.
The authors wish to thank E. Wagner for technical support in data acquisition. Sample fabrication was carried out in the Upstream Nanofabrication Facility (PTA) and CEA LETI MINATEC/DOPT clean rooms. H.A.N. was supported by a Ph.D. scholarship from the Vietnamese government, T.G. by the ANR-QDOT (ANR-16-CE09-0010-01) project, P.L.d.A. by the ANR-WIFO (ANR-11-BS10-011) project and CAPES Young Talents Fellowship Grant No. 88887.059630/2014-00, D.T. by a Ph.D. scholarship from the Rhône-Alpes Region, and N.G. by the Danish Research Council for Technology and Production (Sapere Aude Contract No. DFF-4005-00370).
[1] D. A. Miller, Nat. Photonics 4, 3 (2010).

[2] D. E. Chang, V. Vuletić, and M. D. Lukin, Nat. Photonics 8, 685 (2014).

[3] W. Chen, K. M. Beck, R. Bücker, M. Gullans, M. D. Lukin, H. Tanji-Suzuki, and V. Vuletić, Science 341, 768 (2013).

[4] A. Feizpour, M. Hallaji, G. Dmochowski, and A. M. Steinberg, Nat. Phys. 11, 905 (2015).
[5] Q. A. Turchette, C. J. Hood, W. Lange, H. Mabuchi, and H. J. Kimble, Phys. Rev. Lett. 75, 4710 (1995).

[6] K. M. Birnbaum, A. Boca, R. Miller, A. D. Boozer, T. E. Northup, and H. J. Kimble, Nature (London) 436, 87 (2005).

[7] T. Aoki, A. S. Parkins, D. J. Alton, C. A. Regal, B. Dayan, E. Ostby, K. J. Vahala, and H. J. Kimble, Phys. Rev. Lett. 102, 083601 (2009). 
[8] S. Baur, D. Tiarks, G. Rempe, and S. Dürr, Phys. Rev. Lett. 112, 073901 (2014).

[9] A. Reiserer, N. Kalb, G. Rempe, and S. Ritter, Nature (London) 508, 237 (2014).

[10] T. G. Tiecke, J. D. Thompson, N. P. de Leon, L. R. Liu, V. Vuletić, and M. D. Lukin, Nature (London) 508, 241 (2014).

[11] B. Hacker, S. Welte, G. Rempe, and S. Ritter, Nature (London) 536, 193 (2016).

[12] I. Shomroni, S. Rosenblum, Y. Lovsky, O. Bechler, G. Guendelman, and B. Dayan, Science 345, 903 (2014).

[13] J. Hwang, M. Pototschnig, R. Lettow, G. Zumofen, A. Renn, S. Götzinger, and V. Sandoghdar, Nature (London) 460, 76 (2009).

[14] A. Maser, B. Gmeiner, T. Utikal, S. Götzinger, and V. Sandoghdar, Nat. Photonics 10, 450 (2016).

[15] Q. A. Turchette, R. J. Thomson, and H. J. Kimble, Appl. Phys. B 60, S1 (1995).

[16] A. Auffèves-Garnier, C. Simon, J. M. Gérard, and J. P. Poizat, Phys. Rev. A 75, 053823 (2007).

[17] D. Englund, A. Faraon, I. Fushman, N. Stoltz, P. Petroff, and J. Vučković, Nature (London) 450, 857 (2007).

[18] I. Fushman, D. Englund, A. Faraon, N. Stoltz, P. Petroff, and J. Vučković, Science 320, 769 (2008).

[19] M. T. Rakher, N. G. Stoltz, L. A. Coldren, P. M. Petroff, and D. Bouwmeester, Phys. Rev. Lett. 102, 097403 (2009).

[20] V. Loo, C. Arnold, O. Gazzano, A. Lemaître, I. Sagnes, O. Krebs, P. Voisin, P. Senellart, and L. Lanco, Phys. Rev. Lett. 109, 166806 (2012).

[21] T. Volz, A. Reinhard, M. Winger, A. Badolato, K. J. Hennessy, E. L. Hu, and A. Imamoğlu, Nat. Photonics 6, 605 (2012).

[22] P. Lodahl, S. Mahmoodian, and S. Stobbe, Rev. Mod. Phys. 87, 347 (2015)

[23] S. Sun, H. Kim, G. S. Solomon, and E. Waks, Nat. Nanotechnol. 11, 539 (2016).

[24] L. De Santis, C. Antón, B. Reznychenko, N. Somaschi, G. Coppola, J. Senellart, C. Gómez, A. Lemaître, I. Sagnes, A. G. White, L. Lanco, A. Auffèves, and P. Senellart, Nat. Nanotechnol. 12, 663 (2017).

[25] D. Englund, A. Majumdar, M. Bajcsy, A. Faraon, P. Petroff, and J. Vučković, Phys. Rev. Lett. 108, 093604 (2012).

[26] R. Bose, D. Sridharan, H. Kim, G. S. Solomon, and E. Waks, Phys. Rev. Lett. 108, 227402 (2012).

[27] A. Javadi, I. Söllner, M. Arcari, S. Lindskov Hansen, L. Midolo, S. Mahmoodian, G. Kiršanske, T. Pregnolato, E. H. Lee, J. D. Song, S. Stobbe, and P. Lodahl, Nat. Commun. 6, 8655 (2015).

[28] D. E. Chang, A. S. Sørensen, E. A. Demler, and M. D. Lukin, Nat. Phys. 3, 807 (2007).

[29] P. Kolchin, R. F. Oulton, and X. Zhang, Phys. Rev. Lett. 106, 113601 (2011).
[30] See Supplemental Material at http://link.aps.org/supplemental/ 10.1103/PhysRevB.97.201106 for details on sample geometry, theoretical model, fitting procedure, extra experimental details, and performance of our system in ideal conditions.

[31] A. Majumdar, E. D. Kim, and J. Vùckovíc, Phys. Rev. B 84, 195304 (2011).

[32] A. V. Kuhlmann, J. Houel, D. Brunner, A. Ludwig, D. Reuter, A. D. Wieck, and R. J. Warburton, Rev. Sci. Instrum. 84, 073905 (2013).

[33] J. Bleuse, J. Claudon, M. Creasey, N. S. Malik, J.-M. Gérard, I Maksymov, J.-P. Hugonin, and P. Lalanne, Phys. Rev. Lett. 106, 103601 (2011).

[34] M. Munsch, N. S. Malik, E. Dupuy, A. Delga, J. Bleuse, J. M. Gérard, J. Claudon, N. Gregersen, and J. Mørk, Phys. Rev. Lett. 110, 177402 (2013).

[35] P. Stepanov, A. Delga, N. Gregersen, E. Peinke, M. Munsch, J. Teissier, J. Mørk, M. Richard, J. Bleuse, J. M. Gérard, and J. Claudon, Appl. Phys. Lett. 107, 141106 (2015).

[36] H. A. Nguyen, Ph.D. thesis, Université Grenoble-Alpes, 2016 , https://hal.archives-ouvertes.fr/tel-01360549.

[37] H. J. Carmichael, Statistical Methods in Quantum Optics 1: Master Equations and Fokker-Planck Equations (Springer, Berlin, 2003).

[38] D. Valente, S. Portolan, G. Nogues, J. P. Poizat, M. Richard, J. M. Gérard, M. F. Santos, and A. Auffèves, Phys. Rev. A 85 , 023811 (2012).

[39] S. H. Autler and C. H. Townes, Phys. Rev. 100, 703 (1955).

[40] C. Cohen-Tannoudji, J. Dupont-Roc, and G. Grynberg, AtomPhoton Interactions: Basic Processes and Applications (Wiley, New York, 1992).

[41] X. Xu, B. Sun, P. R. Berman, D. G. Steel, A. S. Bracker, D. Gammon, and L. J. Sham, Science 317, 929 (2007).

[42] G. Jundt, L. Robledo, A. Högele, S. Fält, and A. Imamoğlu, Phys. Rev. Lett. 100, 177401 (2008).

[43] T. Häyrynen, J. R. de Lasson, and N. Gregersen, J. Opt. Soc. Am. A 33, 1298 (2016).

[44] N. Somaschi, V. Giesz, L. De Santis, J. C. Loredo, M. P. Almeida, G. Hornecker, S. L. Portalupi, T. Grange, C. Antón, J. Demory, C. Gómez, I. Sagnes, N. D. Lanzillotti-Kimura, A. Lemaître, A. Auffèves, A. G. White, L. Lanco, and P. Senellart, Nat. Photonics 10, 340 (2016).

[45] A. V. Kuhlmann, J. H. Prechtel, J. Houel, J. A. Ludwig, D. Reuter, A. D. Wieck, and R. J. Warburton, Nat. Commun. 6, 8204 (2015).

[46] N. Gregersen, T. R. Nielsen, J. Mørk, J. Claudon, and J. M. Gérard, Opt. Express 18, 21204 (2010).

[47] M. Makhonin, J. E. Dixon, R. J. Coles, B. Royall, I. J. Luxmoore, E. Clarke, M. Hugues, M. S. Skolnick, and A. M. Fox, Nano Lett. 14, 6997 (2014).

[48] P. Stepanov, A. Delga, X. Zang, J. Bleuse, E. Dupuy, E. Peinke, P. Lalanne, J.-M. Gérard, and J. Claudon, Appl. Phys. Lett. 106, 041112 (2015). 